\title{
SPIN COHERENT-STATE PATH INTEGRALS AND THE INSTANTON CALCULUS
}

\author{
ANUPAM GARG \\ Northwestern University, Department of Physics and Astronomy \\ Evanston, IL 60208 USA \\ EVGUENY KOCHETOV \\ Bogoliubov Theoretical Laboratory, \\ Joint Institute for Nuclear Research, 141980 Dubna, Russia \\ KEE-SU PARK \\ Department of Physics, \\ Pohang University of Science and Technology \\ Pohang, Kyungbuk, Korea \\ MICHAEL STONE \\ University of Illinois, Department of Physics \\ 1110 W. Green St. \\ Urbana, IL 61801 USA
}




\begin{abstract}
We use an instanton approximation to the continuous-time spin coherentstate path integral to obtain the tunnel splitting of classically degenerate ground states. We show that provided the fluctuation determinant is carefully evaluated, the path integral expression is accurate to order $O(1 / j)$. We apply the method to the LMG model and to the molecular magnet $\mathrm{Fe}_{8}$ in a transverse field.
\end{abstract}

This paper is dedicated to the memory of Victor Belinicher, who was lost when Siberia Airlines flight 1812 was shot down over the Black Sea, Oct 4th 2001. Victor made many contributions to physics, in particular to the spin tunneling problem.

PACS numbers: 03.65.Ca, 03.65.Bz

Typeset using REVTEX 


\section{INTRODUCTION}

One of the most convincing demonstrations of quantum effects in a near-macroscopic system is provided by the accurate measurement [1] of the level splittings in the molecular magnet $\mathrm{Fe}_{8}$. These splittings are caused by the quantum tunnelling of the direction of the large $(J=10)$ molecular spin between two classically degenerate energy minima.

The natural tool for studying such spin tunnelling should be the spin $(S U(2))$ coherentstate path integral. It is easy to establish that this formalism gives a good qualitative description of the tunnelling process [2 [4] - including the dramatic topological quenching of the tunnelling [5] that makes the $\mathrm{Fe}_{8}$ results so interesting. Unfortunately, a straightforward application of the spin coherent-state path integral to compute the semiclassical propagator [6] or the tunnel splitting [7] yields results that are incorrect beyond the leading exponential order.

Although there do exist other path integral approaches which find the splitting correctly [8.9], the resulting calculations tend to be intricate, and the simplicity seen in the conventional Schrödinger particle case is lost. These problems have lead to the spin coherent state path integral acquiring a reputation for being mathematically ill defined — or at least harder to deal with than the conventional Feynman path integral, whose mathematical subtleties have been well studied.

Recently, however, it has begun to be appreciated that the problem with the spin coherent state calculation is simply that the fluctuation determinant has an "anomaly", and that, once the "extra phase" provided by the anomaly is taken into account, the coherent state path integral gives correct answers. This extra phase seems to have been originally discovered in the 1980's by Solari [10], but the significance of his result was not widely appreciated. It was then rediscovered by one of the present authors [11] and also by Vieira and Sacramento [12]. The interpretation of the extra phase as an "anomaly" is due to the remaining authors of the present paper [13].

These previous discussions of the extra phase were restricted to the case of quantum 
evolution between generic values of the classical degrees of freedom. However, when we calculate tunnel splitting, the endpoints of the instanton path lie at local minima of the classical energy and, just as in the Schrödinger particle case, the Jacobi fluctuation operator has a zero mode which makes the inverse of its determinant singular and the general formula for the propagator inapplicable. Thus our earlier work had not fully established that the spin coherent state path integral gave the correct result for the tunnel splitting. This we do in the present paper.

In the next section we provide a brief review of the spin coherent-state path integral, including the correction to the fluctuation determinant prefactor. In sections three and four we discuss the complications that ensue when there is a zero mode and provide a general formula for the one-instanton contribution to the tunnelling amplitude. In section five we apply this formula to the relatively simple case of the Lipkin-Meshkov-Glick (LMG) model [14], and in section six we evaluate the tunnel splitting for a realistic model of $\mathrm{Fe}_{8}$.

\section{SPIN COHERENT STATES}

We follow the conventions in [13] and define our spin coherent states [15] to be

$$
|z\rangle=\exp \left(z \hat{J}_{+}\right)|j,-j\rangle
$$

where $|j,-j\rangle$ is the lowest spin state in the $2 j+1$ dimensional representation of $S U(2)$ and $\hat{J}_{+}$is the spin algebra ladder operator obeying

$$
\hat{J}_{+}|j, m\rangle=\sqrt{j(j+1)-m(m+1)}|j, m+1\rangle .
$$

The variable $z$ is a stereographic coordinate on the unit sphere with $z=0$ at the south pole (spin down direction) and $z=\infty$ at the north pole (spin up).

These coherent states are not normalized, but depend holomorphicly on $z$. This means

that matrix elements such as $\left\langle z^{\prime}|\hat{O}| z\right\rangle$ are holomorphic functions of the variable $z$, and antiholomorphic functions of the variable $z^{\prime}$. 
The inner product of two coherent states is

$$
\left\langle z^{\prime} \mid z\right\rangle=\left(1+\bar{z}^{\prime} z\right)^{2 j}
$$

and they satisfy the overcompleteness relation

$$
\mathbf{1}=\frac{2 j+1}{\pi} \int \frac{d^{2} z}{(1+\bar{z} z)^{2 j+2}}|z\rangle\langle z|
$$

Here $d^{2} z$ is shorthand for $d x d y$. The factor $1 /(1+\bar{z} z)^{2}$ combines with this to make the invariant measure on the the two-sphere. The remaining factor in the integration measure, $1 /(1+\bar{z} z)^{2 j}$, serves to normalize the coherent states.

We may use the overcompleteness relation to derive a formal continuous-time path integral representation for the propagator

$$
K\left(\bar{\zeta}_{f}, \zeta_{i}, T\right)=\left\langle\zeta_{f}\left|e^{-i \hat{H} T}\right| \zeta_{i}\right\rangle
$$

We insert $N$ intermediate overcompleteness relations into (2.5) and consider the limit $N \rightarrow$ $\infty$. This leads to the path integration formula [11]

$$
K\left(\bar{\zeta}_{f}, \zeta_{i}, T\right)=\int_{\zeta_{i}}^{\bar{\zeta}_{f}} d \mu(\bar{z}, z) \exp \{S(\bar{z}(t), z(t))\}
$$

where the path measure $d \mu$ is

$$
d \mu(\bar{z}(t), z(t))=\lim _{N \rightarrow \infty} \prod_{n=1}^{N} \frac{2 j+1}{\pi} \frac{d^{2} z_{n}}{\left(1+\bar{z}_{n} z_{n}\right)^{2}}
$$

and the action $S(\bar{z}(t), z(t))$ is

$$
S(\bar{z}(t), z(t))=j\left\{\ln \left(1+\bar{\zeta}_{f} z(T)\right)+\ln \left(1+\bar{z}(0) \zeta_{i}\right)\right\}+\int_{0}^{T}\left\{j \frac{\dot{\bar{z}} z-\bar{z} \dot{z}}{1+\bar{z} z}-i H(\bar{z}, z)\right\} d t
$$

The c-number Hamiltonian, $H(\bar{z}, z)$, is obtained from the operator $\hat{H}$ by

$$
H(\bar{z}, z)=\langle z|\hat{H}| z\rangle /\langle z \mid z\rangle \text {. }
$$

The paths $z(t), \bar{z}(t)$ obey the boundary conditions $z(0)=\zeta_{i}, \bar{z}(T)=\bar{\zeta}_{f}$, but $\bar{z}(0), z(T)$, being actually $\bar{z}(0+\epsilon)$ and $z(T-\epsilon)$, are unconstrained, and are to be integrated over [11]. 
The manipulations leading to the continuous time path integral are heuristic, but with careful treatment the formal path integral should be as useful as the familiar configuration space Feynman path integral. In particular the semiclassical, or large $j$, propagator can be obtained from a stationary phase approximation to the path integral [13].

The stationary phase approximation requires us to seek "classical" trajectories for which $S$ remains stationary as we vary the functions $z(t)$ and $\bar{z}(t)$. These stationary paths will generally be complex. If we write $z$ as $x+i y$ and $\bar{z}=x-i y$, then, except in special cases, $x$ and $y$ are not real numbers. In particular there is no requirement that $\bar{z}(0)$ be the complex conjugate of $z(0) \equiv \zeta_{i}$, nor that $z(T)$ be the complex conjugate of $\bar{z}(T) \equiv \bar{\zeta}_{f}$. Bearing this in mind, we make variations about a chosen path, and keep track of all boundary contributions resulting from integrations by parts. We find that

$$
\begin{aligned}
\delta S= & \frac{2 j z(T)}{1+\bar{\zeta}_{f} z(T)} \delta \bar{z}(T)+\frac{2 j \bar{z}(0)}{1+\bar{z}(0) \zeta_{i}} \delta z(0) \\
& +\int_{0}^{T}\left\{\delta z(t)\left(\frac{2 j \dot{\bar{z}}}{(1+\bar{z} z)^{2}}-i \frac{\partial H}{\partial z}\right)+\delta \bar{z}(t)\left(-\frac{2 j \dot{z}}{(1+\bar{z} z)^{2}}-i \frac{\partial H}{\partial \bar{z}}\right)\right\} d t .
\end{aligned}
$$

Demanding that this change in the action be zero requires the trajectory to obey Hamilton's equations

$$
\dot{\bar{z}}=i \frac{(1+\bar{z} z)^{2}}{2 j} \frac{\partial H}{\partial z}, \quad \dot{z}=-i \frac{(1+\bar{z} z)^{2}}{2 j} \frac{\partial H}{\partial \bar{z}}
$$

together with the conditions $\delta z(0)=0$ and $\delta \bar{z}(T)=0$. We can therefore impose the boundary conditions $z(0)=\zeta_{i}, \bar{z}(T)=\bar{\zeta}_{f}$, but $\bar{z}(0)$ and $z(T)$ are free to vary, and so are determined by the equations of motion. This is important because Hamilton's equations are first order in time and we cannot simultaneously impose initial and final conditions on their solutions.

The dynamically determined endpoints can also be read off from the Hamilton-Jacobi relations that follow from (2.10). These are

$$
\frac{\partial S_{\mathrm{cl}}}{\partial \bar{\zeta}_{f}}=\frac{2 j z(T)}{1+\bar{\zeta}_{f} z(T)}, \quad \frac{\partial S_{\mathrm{cl}}}{\partial \zeta_{i}}=\frac{2 j \bar{z}(0)}{1+\bar{z}(0) \zeta_{i}} .
$$

The Hamilton-Jacobi relations also tell us that 


$$
\frac{\partial S_{\mathrm{cl}}}{\partial \bar{\zeta}_{i}}=\frac{\partial S_{\mathrm{cl}}}{\partial \zeta_{f}}=0
$$

showing that $S_{\mathrm{cl}}$ is a holomorphic function of $\zeta_{i}$, and an anti-holomorphic function of $\zeta_{f}$. These analyticity properties of $S_{\mathrm{cl}}$ coincide with those of $K$. This is reasonable since $\exp S_{\mathrm{cl}}$ is the leading approximation to $K$, and we would expect analyticity to be preserved termby-term in the large $j$ expansion. Finally, we have the Hamilton-Jacobi equation

$$
\frac{\partial S_{\mathrm{cl}}}{\partial T}=-i H\left(\bar{\zeta}_{f}, z(T)\right)
$$

In 113 we showed that after we compute the Gaussian integral over small fluctuations about the stationary phase path the resulting semiclassical approximation to the propagator is

$$
K_{\mathrm{scl}}\left(\bar{\zeta}_{f}, \zeta_{i}, T\right)=\left(\frac{\left(1+\bar{\zeta}_{f} z(T)\right)\left(1+\bar{z}(0) \zeta_{i}\right)}{2 j} \frac{\partial^{2} S_{\mathrm{cl}}}{\partial \zeta_{i} \partial \bar{\zeta}_{f}}\right)^{\frac{1}{2}} \exp \left\{S_{\mathrm{cl}}\left(\bar{\zeta}_{f}, \zeta_{i}, T\right)+\frac{i}{2} \int_{0}^{T} \phi_{\mathrm{SK}}(t) d t\right\}
$$

or a sum of such terms over a set of contributing classical paths. In this expression

$$
\phi_{\mathrm{SK}}(\bar{z}, z)=\frac{1}{2}\left(\frac{\partial}{\partial \bar{z}} \frac{(1+\bar{z} z)^{2}}{2 j} \frac{\partial H}{\partial z}+\frac{\partial}{\partial z} \frac{(1+\bar{z} z)^{2}}{2 j} \frac{\partial H}{\partial \bar{z}}\right)
$$

is the "extra-phase" discovered by Solari, Kochetov, and Vieira and Sacramento.

The form (2.15) is valid only if the prefactor is finite. When we compute instanton contributions to tunnelling there is a zero mode in the quadratic form for small fluctuations, and the resulting divergent integral over this mode is to be replaced by an integral over a collective coordinate labeling the instant that the tunnelling event occurred. This we will describe in the next section.

\section{DEALING WITH THE ZERO MODE}

As is usual in calculating tunnelling effects, it is convenient to perform the computations in Euclidean (imaginary) time. For the sake of symmetry we will take the time evolution as running from $-T / 2$ to $T / 2$ and the propagator (2.15) becomes 


$$
K\left(\bar{\zeta}_{f}, \zeta_{i}, T\right)=[D(T)]^{-\frac{1}{2}} \exp \left\{S_{\mathrm{cl}}+\frac{1}{2} \int_{-T / 2}^{T / 2} \phi_{\mathrm{SK}} d \tau\right\}
$$

where again $\phi_{\mathrm{SK}}$ is the integrand of the Solari-Kochetov phase

$$
\phi_{\mathrm{SK}}=\frac{1}{2}\left(\frac{\partial}{\partial \bar{z}} \frac{(1+\bar{z} z)^{2}}{2 j} \frac{\partial H}{\partial z}+\frac{\partial}{\partial z} \frac{(1+\bar{z} z)^{2}}{2 j} \frac{\partial H}{\partial \bar{z}}\right),
$$

evaluated along $z_{\mathrm{cl}}(\tau), \bar{z}_{\mathrm{cl}}(\tau)$, and $D(T)$ is the fluctuation determinant. The latter may be found by the "shooting method". As explained in [13], this involves solving the equation

$$
\hat{L} \Psi_{L} \equiv\left[\begin{array}{cc}
B(\tau) & -\partial_{\tau}+A(\tau) \\
\partial_{\tau}+A(\tau) & \bar{B}(\tau)
\end{array}\right]\left(\begin{array}{l}
\psi_{L} \\
\bar{\psi}_{L}
\end{array}\right)=0,
$$

where

$$
\begin{aligned}
A=\phi_{\mathrm{SK}} & =\frac{1}{2}\left(\frac{\partial}{\partial \bar{z}} \frac{(1+\bar{z} z)^{2}}{2 j} \frac{\partial H}{\partial z}+\frac{\partial}{\partial z} \frac{(1+\bar{z} z)^{2}}{2 j} \frac{\partial H}{\partial \bar{z}}\right) \\
B & =\frac{\partial}{\partial \bar{z}} \frac{(1+\bar{z} z)^{2}}{2 j} \frac{\partial H}{\partial \bar{z}} \\
\bar{B} & =\frac{\partial}{\partial z} \frac{(1+\bar{z} z)^{2}}{2 j} \frac{\partial H}{\partial z}
\end{aligned}
$$

with the initial condition

$$
\Psi_{L}(-T / 2)=\left(\begin{array}{l}
\psi_{L} \\
\bar{\psi}_{L}
\end{array}\right)_{-T / 2}=\left(\begin{array}{l}
0 \\
1
\end{array}\right)
$$

Given the solution of this equation, we read off the determinant as $D(T)=\bar{\psi}_{L}(T / 2)$. In real time, and when there are no problems with zero-modes, this recipe leads to the prefactor appearing in (2.15).

Now assume that the coherent states $\left|z_{i}\right\rangle$ and $\left|z_{f}\right\rangle$ represent spins pointing along the directions of two equal-energy global minima of the Hamiltonian $\hat{H}$. Because the gradient of the energy vanishes at both ends, the classical path joining $z_{i}$ to $z_{f}$ has the character of an instanton: as the total time taken to traverse the path becomes longer and longer most of the motion still takes place in an "instant", a fixed period short in duration compared to the total. When $T$ becomes infinite, the epoch of this "instant" is arbitrary and this leads to a zero-eigenvalue mode in the fluctuation operator. Thus $D(T)$ is formally zero. 
The problem of dividing by the square root of zero is avoided by introducing a collective coordinate for the tunnelling epoch, and the formal infinity in the one-instanton contribution to the propagator becomes a factor of $T$.

The classical instanton solution can be written $z_{\mathrm{cl}}\left(\tau-\tau_{0}\right), \bar{z}_{\mathrm{cl}}\left(\tau-\tau_{0}\right)$ where $\tau_{0}$ is the epoch at which the tunnelling occurs. Since, in the large $T$ limit, the action for the tunnelling event is independent of $\tau_{0}$, the normalized zero mode is

$$
\Psi_{0}=\left(\begin{array}{c}
\psi_{0}(\tau) \\
\bar{\psi}_{0}(\tau)
\end{array}\right)=\frac{\sqrt{g}}{1+\bar{z}_{\mathrm{cl}} z_{\mathrm{cl}}}\left(\begin{array}{c}
\dot{z}_{\mathrm{cl}}(\tau) \\
\dot{\bar{z}}_{\mathrm{cl}}(\tau)
\end{array}\right),
$$

where $g$ is chosen to make

$$
\int_{-T / 2}^{T / 2} \Psi_{0}^{t} \Psi_{0} d \tau=\int_{-T / 2}^{T / 2}\left(\psi_{0}^{2}+\bar{\psi}_{0}^{2}\right) d \tau=1 .
$$

The divergent Gaussian integration over the coefficient of the zero mode is replaced by an integral over possible tunnelling epochs $\tau_{0}$ by inserting a factor of

$$
1=\frac{1}{\sqrt{2 \pi \alpha}} \int_{-T / 2}^{T / 2} d \tau_{0}\left(\frac{\partial \mathcal{F}}{\partial \tau_{0}}\right) \exp -\frac{1}{2 \alpha} \mathcal{F}^{2}\left(\tau_{0}\right)
$$

into the path integral, with the choice

$$
\mathcal{F}\left(\tau_{0}\right)=\int_{-T / 2}^{T / 2} d \tau^{\prime} \frac{1}{1+\bar{z}_{\mathrm{cl}} z_{\mathrm{cl}}\left(\tau^{\prime}-\tau_{0}\right)} \Psi_{0}^{t}\left(\tau^{\prime}-\tau_{0}\right)\left(\begin{array}{c}
z\left(\tau^{\prime}\right) \\
\bar{z}\left(\tau^{\prime}\right)
\end{array}\right),
$$

and then proceeding in a manner similar to that used for quantum mechanical instantons in the Feynman path integral [16,17]: we first set $z=z_{\mathrm{cl}}\left(\tau-\tau_{0}\right)+\delta z\left(\tau-\tau_{0}\right)$ and similarly $\bar{z}$. Next, after observing that everything depends only on the combination $\tau-\tau_{0}$, we change variables $\tau-\tau_{0} \rightarrow \tau$. The integral over $\tau_{0}$ is then trivial and gives a factor of $T$. Meanwhile, after an integration by parts and ignoring the fuctuations of $(z, \bar{z})$ about $\left(z_{\mathrm{cl}}, \bar{z}_{\mathrm{cl}}\right)$ which are of higher order, the Jacobian factor becomes

$$
\frac{\partial \mathcal{F}}{\partial \tau_{0}}=\int_{-T / 2}^{T / 2} d \tau^{\prime} \Psi_{0}^{t} \frac{1}{1+\bar{z}_{\mathrm{cl}} z_{\mathrm{cl}}}\left(\begin{array}{c}
\dot{z}_{\mathrm{cl}}\left(\tau^{\prime}\right) \\
\dot{\bar{z}}_{\mathrm{cl}}\left(\tau^{\prime}\right)
\end{array}\right)=\frac{1}{\sqrt{g}} .
$$

The quadratic term in the exponent is a projector onto the zero mode and replaces the vanishing eigenvalue by $1 / 2 j \alpha$. The net result is the replacement 


$$
[D(T)]^{-\frac{1}{2}} \rightarrow T \sqrt{\frac{j}{\pi g}}\left[\frac{D(T)}{\lambda_{0}}\right]^{-\frac{1}{2}},
$$

where $\lambda_{0}(T)$ is the eigenvalue that vanishes as $T$ becomes large.

The desired ratio, $\operatorname{Det}^{\prime}(\hat{L})=D(T) / \lambda_{0}$, is equal to $\bar{\psi}_{L}(T / 2) / \lambda_{0}(T)$. We will not have to obtain $\bar{\psi}_{L}(T / 2)$ and $\lambda_{0}(T)$ separately.

The eigenvalue problem is

$$
\hat{L} \Psi_{\lambda}=\lambda \Psi_{\lambda} ; \quad \Psi_{\lambda}=\left(\begin{array}{c}
\psi_{\lambda} \\
\bar{\psi}_{\lambda}
\end{array}\right)
$$

where $\hat{L}$ is the same operator as in (3.3), but with boundary conditions $\psi_{\lambda}(-T / 2)=$ $\bar{\psi}_{\lambda}(T / 2)=0$.

For finite $T$ the shooting method solution, $\Psi_{L}$, is close to, but not quite equal to, the "small-eigenvalue" eigenfunction, $\Psi_{\lambda_{0}}$. Although $\Psi_{L}$ obeys the boundary condition at $\tau=$ $-T / 2$, it does not quite obey the boundary condition at $\tau=+T / 2$. In turn $\Psi_{\lambda_{0}}$ is close to, but not quite equal to, the infinite- $T$ zero-eigenvalue mode, $\Psi_{0}$.

Now $\Psi_{0}$ obeys the equation $\hat{L} \Psi_{0}=0$, but no particular boundary conditions at $\pm T / 2$. There is a second solution of this equation, $\Xi_{0}=\left(\xi_{0}, \bar{\xi}_{0}\right)^{t}$. The Wronskian of these solutions

$$
W(\Psi, \Xi)=\left|\begin{array}{ll}
\psi_{0}(\tau) & \xi_{0}(\tau) \\
\bar{\psi}_{0}(\tau) & \bar{\xi}_{0}(\tau)
\end{array}\right|
$$

is independent of $\tau$. Next we observe that the differential equation (3.12) can be converted to an integral equation

$$
\begin{aligned}
\Psi_{\lambda}(\tau) & =\Psi_{L}(\tau)+\lambda \int_{-T / 2}^{\tau} d \tau^{\prime} G\left(\tau, \tau^{\prime}\right) \Psi_{\lambda}\left(\tau^{\prime}\right), \\
& =\Psi_{L}(\tau)+\frac{\lambda}{W} \int_{-T / 2}^{\tau} d \tau^{\prime}\left[\Psi_{0}(\tau) \Xi_{0}^{t}\left(\tau^{\prime}\right)-\Xi_{0}(\tau) \Psi_{0}^{t}\left(\tau^{\prime}\right)\right] \Psi_{\lambda}\left(\tau^{\prime}\right) .
\end{aligned}
$$

Since $\Psi_{L}(\tau)$ obeys the boundary conditions at $-T / 2$, and the integral vanishes at this point, we can find the eigenvalues $\lambda$ by requiring that the lower component at of $\Psi_{\lambda}$ vanishes at $\tau=T / 2$. We are only interested in solutions where $\lambda=\lambda_{0}$ is very small. Because of this we can approximate the $\Psi_{\lambda}\left(\tau^{\prime}\right)$ appearing in the integral in (3.14) by the zeroth-order solution, $\Psi_{L}$. In this way we see that 


$$
\frac{\bar{\psi}_{L}(T / 2)}{\lambda_{0}(T)}=-\frac{1}{W} \int_{-T / 2}^{T / 2} d \tau\left[\bar{\psi}_{0}(T / 2) \Xi_{0}^{t}(\tau)-\bar{\xi}_{0}(T / 2) \Psi_{0}^{t}(\tau)\right] \Psi_{L}(\tau)
$$

The integral in (3.15) may be evaluated using only the asymptotic behaviour of $\Psi_{0}$ and $\Xi_{0}$, which involve $z_{\mathrm{cl}}$ and $\bar{z}_{\mathrm{cl}}$. This asymptotic behaviour depends only on the form of the Hamiltonian in the neighbourhood of the endpoints.

In all cases we consider the instanton solutions have the property that $\bar{z}_{\mathrm{cl}}=z_{\mathrm{cl}}^{*}$ at their endpoints. Here the asterisk denotes a true complex conjugate as opposed to the formal conjugate denoted by the bar. The coincidence of the formal and true conjugate occurs because these endpoints lie on the real unit sphere't. Taking this observation into account, we parametrize the Hamiltonian in the vicinity of the initial stationary point in terms of two frequencies, $\omega_{1,2}$, as

$$
H(\bar{z}, z) \approx \frac{2 j}{\left(1+z_{i}^{*} z_{i}\right)^{2}}\left[\omega_{1}\left(z-z_{i}\right)\left(\bar{z}-z_{i}^{*}\right)+\frac{1}{2} \omega_{2}\left(z-z_{i}\right)^{2}+\frac{1}{2} \omega_{2}^{*}\left(\bar{z}-z_{i}^{*}\right)^{2}\right] .
$$

Since $H(\bar{z}, z)$ is real, so is $\omega_{1}$. Also, because the initial point is an energy minimum, we must have $\omega_{1}>\left|\omega_{2}\right|$. We can therefore define a real mean frequency, $\omega$, by

$$
\omega^{2} \equiv \omega_{1}^{2}-\omega_{2} \omega_{2}^{*}
$$

A similar expression holds at $z_{f}$ with the same values of $\omega_{1}$ and $\omega_{2}$ provided the degeneracy in the Hamiltonian is due to some symmetry. (There might be an extra phase factor in $\omega_{2}$, but this makes no difference to the subsequent calculation).

As $\tau$ becomes large and negative, $B \rightarrow \omega_{2}, \bar{B} \rightarrow \omega_{2}^{*}$ and $A=\phi_{S K} \rightarrow \omega_{1}$, so we see that

$$
\left(\begin{array}{l}
\psi_{0} \\
\bar{\psi}_{0}
\end{array}\right) \rightarrow\left(\begin{array}{l}
\psi_{0-} \\
\bar{\psi}_{0-}
\end{array}\right) e^{\omega \tau} ; \quad\left(\begin{array}{l}
\xi_{0} \\
\bar{\xi}_{0}
\end{array}\right) \rightarrow\left(\begin{array}{l}
\xi_{0-} \\
\bar{\xi}_{0-}
\end{array}\right) e^{-\omega \tau},
$$

where

\footnotetext{
${ }^{1}$ This is a not a trivial observation: in a more accurate representation of the $\mathrm{Fe}_{8}$ problem which includes fourth order anisotropy terms there are additional instantons for which this fails to be true.
} 


$$
\left[\begin{array}{cc}
\omega_{2} & -\omega+\omega_{1} \\
\omega+\omega_{1} & \omega_{2}^{*}
\end{array}\right]\left(\begin{array}{l}
\psi_{0-} \\
\bar{\psi}_{0-}
\end{array}\right)=0
$$

There is an analogous relation for $\left(\xi_{0-}, \bar{\xi}_{0-}\right)^{t}$. We can use the Wronskian to connect $\Psi_{0-}$ with $\Xi_{0-}$, so everything can be expressed in terms of $W$ and the normalization $g$. Similar remarks apply to $\Psi_{0+}$ and $\Xi_{0+}$. If we write

$$
\left(\begin{array}{l}
\psi_{L} \\
\bar{\psi}_{L}
\end{array}\right)=\alpha\left(\begin{array}{l}
\psi_{0} \\
\bar{\psi}_{0}
\end{array}\right)+\beta\left(\begin{array}{c}
\xi_{0} \\
\bar{\xi}_{0}
\end{array}\right)
$$

and apply the boundary condition at $-T / 2$ we can find $\alpha$ and $\beta$, and hence

$$
\left(\begin{array}{c}
\psi_{L}(\tau) \\
\bar{\psi}_{L}(\tau)
\end{array}\right)=\frac{1}{W}\left[-\xi_{0-} e^{\omega T / 2}\left(\begin{array}{c}
\psi_{0}(\tau) \\
\bar{\psi}_{0}(\tau)
\end{array}\right)+\psi_{0-} e^{-\omega T / 2}\left(\begin{array}{c}
\xi_{0}(\tau) \\
\bar{\xi}_{0}(\tau)
\end{array}\right)\right]
$$

Inserting this into (3.15) and noting that the $\psi_{0} \bar{\psi}_{0}$ terms dominate, we find

$$
\frac{\bar{\psi}_{L}(T / 2)}{\lambda_{0}(T)}=-\frac{1}{W^{2}} \xi_{0-} \bar{\xi}_{0+} e^{\omega T} \int_{-T / 2}^{T / 2}\left(\psi_{0}^{2}+\bar{\psi}_{0}^{2}\right) d \tau
$$

or,

$$
\frac{\bar{\psi}_{L}(T / 2)}{\lambda_{0}(T)}=\frac{\left|\omega_{2}\right|^{2}}{\psi_{0-} \bar{\psi}_{0+}} \frac{e^{\omega T}}{4 \omega^{2}}
$$

Thus the one-instanton contribution to the propagator is

$$
K\left(\bar{z}_{f}, z_{i}, T\right)=\exp \left\{S_{\mathrm{cl}}+\frac{1}{2} \int_{-T / 2}^{T / 2} \phi_{\mathrm{SK}} d \tau\right\} \sqrt{\frac{j}{\pi g}}\left[\frac{\psi_{0-} \bar{\psi}_{0+}}{\left|\omega_{2}\right|^{2}}\right]^{\frac{1}{2}}\left(2 \omega T e^{-\frac{1}{2} \omega T}\right) .
$$

Note that $\psi_{0}, \bar{\psi}_{0}$ are proportional to $\sqrt{g}$, thus $\sqrt{g}$ drops out and we can simply put $g=1$ in the sequel.

Let

$$
\begin{gathered}
\dot{z}_{\mathrm{cl}} \approx \omega \zeta_{-} e^{\omega \tau}, \quad \tau \rightarrow-\infty \\
\dot{\bar{z}}_{\mathrm{cl}} \approx \omega \bar{\zeta}_{+} e^{-\omega \tau}, \quad \tau \rightarrow+\infty
\end{gathered}
$$

then

$$
\psi_{0-} \bar{\psi}_{0+}=\frac{\omega^{2} \zeta_{-} \bar{\zeta}_{+}}{N}
$$


with

$$
N=\left(1+\bar{z}_{i} z_{i}\right)\left(1+\bar{z}_{f} z_{f}\right)
$$

Using this we can write

$$
K\left(\bar{z}_{f}, z_{i}, T\right)=\exp \left\{S_{\mathrm{cl}}+\frac{1}{2} \int_{-T / 2}^{T / 2} \phi_{\mathrm{SK}} d \tau\right\} \sqrt{\frac{j}{\pi N}}\left[\frac{\bar{\zeta}_{+} \zeta_{-}}{\left|\omega_{2}\right|^{2}}\right]^{\frac{1}{2}}\left(2 \omega^{2} T e^{-\frac{1}{2} \omega T}\right)
$$

\section{EXTRACTING THE ENERGY SPLITTING}

Again assume that the coherent states $\left|z_{i}\right\rangle$ and $\left|z_{f}\right\rangle$ represent spins pointing along the directions of two equal energy global minima of the Hamiltonian $\hat{H}$. Let $\left|\psi_{i, f}\right\rangle$ be the approximate (tunnelling-ignored) energy eigenstates localized near these minima. These should have their phases chosen so that when tunnelling is included the eigenstates become the linear combinations

$$
\left|\psi_{ \pm}\right\rangle=\frac{1}{\sqrt{2}}\left(\left|\psi_{i}\right\rangle \pm\left|\psi_{f}\right\rangle\right)
$$

If the energies of these states are

$$
E_{ \pm}=E_{\mathrm{av}} \pm \frac{1}{2} \Delta
$$

and $a_{\alpha} \equiv\left\langle z_{\alpha} \mid \psi_{\alpha}\right\rangle$, then as $T$ becomes large the coherent-state propagator

$$
K\left(\bar{z}_{f}, z_{i}, T\right)=\left\langle z_{f}\left|e^{-\hat{H} T}\right| z_{i}\right\rangle,
$$

is given by

$$
\begin{aligned}
K\left(\bar{z}_{f}, z_{i}, T\right) & \approx a_{f} a_{i}^{*} e^{-E_{\mathrm{av}} T} \sinh \left(\frac{1}{2} \Delta T\right) \\
& =a_{f} a_{i}^{*} e^{-E_{\mathrm{av}} T}\left(\frac{1}{2} \Delta T+\frac{1}{6} \frac{\Delta^{3} T^{3}}{2^{3}}+\cdots\right) .
\end{aligned}
$$

We will find the energy splitting, $\Delta$, by evaluating $K$ in the one-instanton approximation and comparing with this expression. 
It is necessary to find expressions for the amplitudes $a_{i}$ and $a_{f}$. These are obtained by looking at

$$
K_{f}=\left\langle z_{f}\left|e^{-\hat{H} T}\right| z_{f}\right\rangle \approx\left|a_{f}\right|^{2} e^{-E_{\mathrm{av}} T}
$$

and

$$
K_{i}=\left\langle z_{i}\left|e^{-\hat{H} T}\right| z_{i}\right\rangle \approx\left|a_{i}\right|^{2} e^{-E_{\mathrm{av}} T}
$$

both evaluated in the harmonic approximation. This evaluation is performed in the appendix. This results in

$$
K_{f}=\left(1+\bar{z}_{f} z_{f}\right)^{2 j} \sqrt{\frac{2 \omega}{\omega+\omega_{1}}} e^{-\frac{1}{2}\left(\omega-\omega_{1}\right) T}
$$

and a similar expression for $K_{i}$. Thus

$$
\frac{1}{2} \Delta=\frac{e^{S_{\mathrm{cl}}+\frac{1}{2} \int_{-T / 2}^{T / 2}\left(\phi_{\mathrm{SK}}-\omega_{1}\right) d \tau}}{\left[\left(1+\bar{z}_{f} z_{f}\right)^{j}\left(1+\bar{z}_{i} z_{i}\right)^{j}\right]} \sqrt{\frac{j}{\pi N}}\left[2 \omega\left(\omega+\omega_{1}\right)\right]^{\frac{1}{2}} \omega\left[\frac{\bar{\zeta}_{+} \zeta_{-}}{\left|\omega_{2}\right|^{2}}\right]^{\frac{1}{2}} .
$$

Now

$$
\frac{2 \omega\left(\omega+\omega_{1}\right)}{\omega_{2}^{2}}=\frac{2 \omega}{\omega_{1}-\omega}
$$

so finally

$$
\Delta=2 \omega \sqrt{P} e^{I}
$$

where

$$
P=\frac{j}{\pi N} \frac{2 \omega}{\omega_{1}-\omega} \bar{\zeta}_{+} \zeta_{-}
$$

and

$$
I=j \int_{-\infty}^{\infty} a_{\mathrm{wZ}}(\tau) d \tau+\frac{1}{2} \int_{-\infty}^{\infty}\left(\phi_{\mathrm{SK}}-\omega_{1}\right) d \tau
$$

where $a_{\mathrm{wz}}$ is the kinetic term

$$
a_{\mathrm{WZ}}(\tau)=\frac{\dot{\bar{z}}_{\mathrm{cl}} z_{\mathrm{cl}}-\dot{z}_{\mathrm{cl}} \bar{z}_{\mathrm{cl}}}{1+\bar{z}_{\mathrm{cl}} z_{\mathrm{cl}}}
$$

in the classical action - the boundary terms having cancelled with the $\left(1+\bar{z}_{f} z_{f}\right)^{j}\left(1+\bar{z}_{i} z_{i}\right)^{j}$ in the denominator. 


\section{THE LMG MODEL.}

In this section we will evaluate the tunnel splitting in the relatively simple case of the Lipkin-Meshkov-Glick (LMG) model [14.

We will take the LMG Hamiltonian to be

$$
\hat{H}=\frac{w}{\sqrt{2}(2 j-1)}\left(\hat{J}_{+}^{2}+\hat{J}_{-}^{2}\right)+\frac{j w}{\sqrt{2}},
$$

with $w>0$. Since $\hat{J}_{+}^{2}+\hat{J}_{-}^{2}=2\left(\hat{J}_{x}^{2}-\hat{J}_{y}^{2}\right)$, we see that the classical minima lie along $\pm \hat{y}$. The Hamiltonian which appears in the path integral is

$$
H(\bar{z}, z)=\frac{\langle z|\hat{H}| z\rangle}{\langle z \mid z\rangle}=\sqrt{2} j w \frac{z^{2}+\bar{z}^{2}}{(1+\bar{z} z)^{2}}+\frac{j w}{\sqrt{2}} .
$$

By setting $\partial H / \partial z=\partial H / \partial \bar{z}=0$, the classical minima are found to be at the points

$$
(z, \bar{z})=(i,-i), \quad(-i, i)
$$

which correspond to the $\pm \hat{y}$ directions of the Cartesian axes. The explicitly added constant in $\hat{H}$ is chosen to make $H(\bar{z}, z)$ zero at these points.

Now we write down the equations of motion for the instantons

$$
\begin{aligned}
& \dot{\bar{z}}=\sqrt{2} w \frac{z-\bar{z}^{3}}{(1+\bar{z} z)}, \\
& \dot{z}=-\sqrt{2} w \frac{\bar{z}-z^{3}}{(1+\bar{z} z)} .
\end{aligned}
$$

We seek a solution which goes from $\left(z_{i}, \bar{z}_{i}\right)=(-i, i)$ to $\left(z_{f}, \bar{z}_{f}\right)=(i,-i)$. The two equations in (5.4) can be decoupled by exploiting the energy conservation condition $H(\bar{z}, z)=0$ which follows from the Hamiltonian nature of the trajectory. This can be written as

$$
2\left(z^{2}+\bar{z}^{2}\right)+1+2 \bar{z} z+\bar{z}^{2} z^{2}=0
$$

and may be solved to yield $z$ as a function of $\bar{z}$ and vice versa:

$$
\bar{z}=-i \frac{\sqrt{2} z+i}{z+\sqrt{2} i}, \quad z=-i \frac{\sqrt{2} \bar{z}+i}{\bar{z}+\sqrt{2} i} .
$$


(Choosing the other solution of the quadratic equation yields instantons running in the opposite direction.) Substituting these formulae in the equations of motion yields

$$
\dot{\bar{z}}=-i w\left(1+\bar{z}^{2}\right), \quad \dot{z}=i w\left(1+z^{2}\right)
$$

These may be integrated by elementary means to yield

$$
\begin{aligned}
& z_{\mathrm{cl}}(\tau)=i \frac{e^{2 w \tau}-C}{e^{2 w \tau}+C}=i \tanh w\left(\tau-\tau_{0}\right), \\
& \bar{z}_{\mathrm{cl}}(\tau)=-i \frac{e^{2 w \tau}-C^{\prime}}{e^{2 w \tau}+C^{\prime}}=-i \tanh w\left(\tau-\tau_{0}^{\prime}\right),
\end{aligned}
$$

where $C=e^{2 w \tau_{0}}, C^{\prime}=e^{2 w \tau_{0}^{\prime}}$. These constants are not independent. Energy conservation requires

$$
\frac{C^{\prime}}{C}=\frac{\sqrt{2}-1}{\sqrt{2}+1}
$$

It is useful at this point to find the frequencies $\omega, \omega_{1}$ and $\omega_{2}$. We have

$$
\omega_{1}=\left.\frac{\left(1+\bar{z}_{i} z_{i}\right)^{2}}{2 j} \frac{\partial^{2} H}{\partial z \partial \bar{z}}\right|_{i}, \quad \omega_{2}=\left.\frac{\left(1+\bar{z}_{i} z_{i}\right)^{2}}{2 j} \frac{\partial^{2} H}{\partial z^{2}}\right|_{i}
$$

where the suffix $i$ means that the derivatives are to be evaluated at the initial point. Carrying out the algebra, we obtain

$$
\omega_{1}=\frac{3}{\sqrt{2}} w, \quad \omega_{2}=\frac{1}{\sqrt{2}} w
$$

Hence,

$$
\omega=\left(\omega_{1}^{2}-\omega_{2}^{2}\right)^{1 / 2}=2 w
$$

We can now evaluate the Wess-Zumino and Solari-Kochetov terms in the tunnelling action. We denote these by $I_{\mathrm{WZ}}$ and $I_{\mathrm{SK}}$ respectively. We begin with $I_{\mathrm{WZ}}$. If we make use of Eq. (5.7), we find

$$
a_{\mathrm{WZ}}(\tau)=\frac{1}{1+\bar{z} z}(\dot{\bar{z}} z-\bar{z} \dot{z})=-i w(\bar{z}+z)
$$

Substituting the explicit forms and performing the integration we get 


$$
I_{\mathrm{WZ}}=-j \ln \left(C / C^{\prime}\right)=-2 j \ln (1+\sqrt{2}) .
$$

Now we integrate the Solari-Kochetov term along the instanton trajectory. We find that

$$
\phi_{\mathrm{SK}}=-\sqrt{2} \frac{\left(z^{2}+\bar{z}^{2}\right)(2+\bar{z} z)}{(1+\bar{z} z)^{2}} .
$$

By energy conservation this equals

$$
\frac{w}{\sqrt{2}}(2+\bar{z} z)
$$

Thus, along the instanton,

$$
\phi_{\mathrm{SK}}-\omega_{1}=-\frac{w}{\sqrt{2}}(1-\bar{z} z)=-i w(\bar{z}+z) .
$$

Hence

$$
I_{\mathrm{SK}}=\frac{1}{2} \int_{-\infty}^{\infty}\left(\phi_{\mathrm{SK}}-\omega_{1}\right) d \tau=-\ln (1+\sqrt{2}) .
$$

The total tunnelling action is

$$
I=-(2 j+1) \ln (1+\sqrt{2})
$$

The shift $2 j \rightarrow 2 j+1$ is due to the Solari-Kochetov correction.

We must now evaluate $P$. This consists of a product of various factors, all of which are to hand. Thus,

$$
\frac{j}{\pi N}=\frac{j}{4 \pi}
$$

The factors $\bar{\zeta}_{+}$and $\zeta_{-}$are found by differentiating the formulas (5.8) and (5.9) and examining the limits $\tau \rightarrow \pm \infty$. In this we way we get

$$
\zeta_{-} \bar{\zeta}_{+}=4 \frac{C^{\prime}}{C} .
$$

Finally,

$$
\frac{2 \omega}{\omega_{1}-\omega}=4 \sqrt{2}(3+2 \sqrt{2}) .
$$


Putting these together, we obtain

$$
P=-\frac{4 j}{\pi}(4+3 \sqrt{2}) \frac{C^{\prime}}{C}=\frac{4 j}{\pi} \sqrt{2}
$$

At this point we have almost all that we need to write down the answer for the tunnel splitting - except that we need to consider a second instanton. The trajectory (5.8) and (5.9) passes close to the north pole of the sphere. By symmetry there must be a second instanton which passes near the south pole. This is given by

$$
z_{\mathrm{cl}}=i \operatorname{coth} w\left(\tau-\tau_{0}\right), \quad \bar{z}_{\mathrm{cl}}=-i \operatorname{coth} w\left(\tau-\tau_{0}^{\prime}\right)
$$

It is obvious by symmetry again that this instanton has exactly the same amplitude as the first, so the total amplitude (and thus the splitting) is obtained by simply doubling the answer from the first instanton. Hence

$$
\Delta=16 w\left(\frac{j}{\pi}\right)^{1 / 2} 2^{1 / 4} e^{-(2 j+1) \ln (1+\sqrt{2})} .
$$

This agrees with [9,8, 18] [In the last reference put $\xi^{2}=1 / \sqrt{2}$ in Eqs. 4.31-4.34.] In [18 there a numerical comparison which shows that the prefactor is indeed correct.

For completeness, we note that the average energy is given by $E_{\mathrm{av}}=\frac{1}{2}\left(\omega-\omega_{1}\right)$.

\section{APPLICATION TO FE 8}

The LMG model is of interest primarily because it provides a check of our formalism against other well-confirmed calculations. In this section we will calculate the tunnel splitting for a family of models that includes a realistic approximation to the molecular magnet $\mathrm{Fe}_{8}$. The spin-direction dependent energy in $\mathrm{Fe}_{8}$ is less symmetric than that of the LMG, and the relevant Hamiltonian includes an externally imposed magnetic field which serves to pull the classical minima off the equator of the unit sphere. It is the experimentally observed oscillations in the tunnel splitting as a function of the external field that makes this system interesting. The oscillations are a consequence of interference between the two distinct instanton trajectories and are accurately reproduced by our calculation. 
We take as our Hamiltonian

$$
\hat{H}=k_{1} \hat{J}_{z}^{2}+k_{2} \hat{J}_{y}^{2}-g \mu_{B} H \hat{J}_{z}
$$

with $k_{1}>k_{2}>0$. We define $\lambda=k_{2} / k_{1}, H_{c}=2 k_{1} j / g \mu_{B}$ and

$$
h=H / H_{c} .
$$

We will express all results in terms of the combinations $\lambda$ and $h$. It is also convenient to define a $1 / j$ corrected field $\tilde{h}$, and anisotropy $\tilde{k}_{1}$ by

$$
\tilde{h}=j h /\left(j-\frac{1}{2}\right), \quad \tilde{k}_{1}=k\left(j-\frac{1}{2}\right) / j .
$$

We follow the same steps as in the LMG model. The "classical" Hamiltonian appearing in the path integral is

$$
H(\bar{z}, z)=\frac{\langle z|\hat{H}| z\rangle}{\langle z \mid z\rangle}=\tilde{k}_{1} j^{2}\left[\frac{(1-\bar{z} z)^{2}-\lambda(z-\bar{z})^{2}-2 \tilde{h}\left(1-\bar{z}^{2} z^{2}\right)}{(1+\bar{z} z)^{2}}\right] .
$$

The energy minima are now at the points

$$
\bar{z}=z= \pm z_{0}
$$

where $z_{0}$ is real and given by

$$
z_{0}=[(1-\tilde{h}) /(1+\tilde{h})]^{1 / 2}
$$

In Cartesian coordinates these minima lie in the $x z$ plane — provided we confine ourselves to $\tilde{h}<1$, which we shall do. In fact, we will assume that

$$
\tilde{h}<\sqrt{1-\lambda} \text {. }
$$

At the two minima, the energy is

$$
\epsilon_{0}=H\left(\bar{z}_{0}, z_{0}\right)=-\tilde{k}_{1} j^{2} \tilde{h}^{2} .
$$

The classical equations of motion are 


$$
\begin{aligned}
& \dot{\bar{z}}=\frac{\tilde{k}_{1} j}{(1+\bar{z} z)}\left[-2 \bar{z}(1-\bar{z} z)+\lambda(\bar{z}-z)\left(1+\bar{z}^{2}\right)+2 \tilde{h} \bar{z}(1+\bar{z} z)\right], \\
& \dot{z}=-\frac{\tilde{k}_{1} j}{(1+\bar{z} z)}\left[-2 z(1-\bar{z} z)+\lambda(z-\bar{z})\left(1+z^{2}\right)+2 \tilde{h} z(1+\bar{z} z)\right] .
\end{aligned}
$$

We wish to solve these subject to the boundary conditions $z_{i}=z(-\infty)=z_{0}, \bar{z}_{f}=z(\infty)=$ $-z_{0}$. Note that $\bar{z}_{i}=z_{i}, z_{f}=\bar{z}_{f}$, so the instanton end points still lie on the real sphere, but the rest of the instanton does not. Once again the equations can be decoupled by exploiting the fact that energy is conserved along the instanton trajectory. In this case $H(\bar{z}, z)=\epsilon_{0}$. This condition can be written as

$$
(1-\bar{z} z)^{2}-\lambda(z-\bar{z})^{2}-2 \tilde{h}\left(1-\bar{z}^{2} z^{2}\right)=-\tilde{h}^{2}(1+\bar{z} z)^{2}
$$

and may be solved to give

$$
\bar{z}=\frac{\sqrt{\lambda} z \pm(1-\tilde{h})}{\sqrt{\lambda} \pm(1+\tilde{h}) z} .
$$

Substituting this in the equation of motion for $\dot{z}$, and simplifying, we get

$$
\dot{z}= \pm \sqrt{\lambda}(1+\tilde{h}) \tilde{k}_{1} j\left(z_{0}^{2}-z^{2}\right)
$$

We will see that to obtain instantons going from $z_{0}$ to $-z_{0}$, we must pick the minus sign in this equation. The other sign yields instantons running in the opposite direction.

It is now elementary to integrate Eq. (6.12), and use Eq. (6.11) to obtain the time dependence for both $z_{\mathrm{cl}}(\tau)$ and $\bar{z}_{\mathrm{cl}}(\tau)$. We find

$$
\begin{aligned}
& z_{\mathrm{cl}}(\tau)=-z_{0} \tanh t \\
& \bar{z}_{\mathrm{cl}}(\tau)=-z_{0} \frac{\sqrt{\lambda} \tanh t+\sqrt{1-\tilde{h}^{2}}}{\sqrt{\lambda}+\sqrt{1-\tilde{h}^{2}} \tanh t} .
\end{aligned}
$$

Here,

$$
t=\omega \tau / 2
$$

and the frequency $\omega$ is given by 


$$
\omega=2 \tilde{k}_{1} j\left[\lambda\left(1-\tilde{h}^{2}\right)\right]^{1 / 2}
$$

That this is the same $\omega$ that follows from Eqs. (3.16) and (3.17) shall be shown shortly. It can be seen that our solution corresponds to choosing the minus sign in Eq. (6.12) as asserted above. It is also useful to note that the solution (6.13) and (6.14) can be rewritten as

$$
z_{\mathrm{cl}}=-z_{0} \tanh t, \quad \bar{z}_{\mathrm{cl}}=-z_{0} \operatorname{coth}\left(t+t_{0}\right),
$$

where

$$
\tanh t_{0}=\left(\frac{\lambda}{1-\tilde{h}^{2}}\right)^{1 / 2}
$$

Equations (6.12) and (6.11) possess a second solution,

$$
z_{\mathrm{cl}}=-z_{0} \operatorname{coth} t, \quad \bar{z}_{\mathrm{cl}}=-z_{0} \tanh \left(t+t_{0}\right)
$$

Formally, this new trajectory can be obtained from the first by the shift $t \rightarrow t+i \pi / 2$. Alternatively, we could obtain it by switching the expressions for $z_{\mathrm{cl}}$ and $\bar{z}_{\mathrm{cl}}$ in Eqs. (6.13) and (6.14), which corresponds to reflection in the $x z$ plane - a symmetry of the Hamiltonian - and then shifting $t$ by $-t_{0}$.

Again we find the frequencies $\omega, \omega_{1}$ and $\omega_{2}$. We note that

$$
\omega_{1}=\left.\frac{\left(1+\bar{z}_{i} z_{i}\right)^{2}}{2 j} \frac{\partial^{2} H}{\partial z \partial \bar{z}}\right|_{i}, \quad \omega_{2}=\left.\frac{\left(1+\bar{z}_{i} z_{i}\right)^{2}}{2 j} \frac{\partial^{2} H}{\partial z^{2}}\right|_{i}
$$

where the suffix $i$ means that the derivatives are to be evaluated at the initial point $\bar{z}=z=$ $z_{i}$. Carrying out the algebra, we obtain

$$
\begin{aligned}
& \omega_{1}=\tilde{k}_{1} j\left(1-\tilde{h}^{2}+\lambda\right), \\
& \omega_{2}=\tilde{k}_{1} j\left(1-\tilde{h}^{2}-\lambda\right) .
\end{aligned}
$$

We now use Eq. (3.17) to show that $\omega$ is given by Eq. (6.16). The same frequencies are found at the final point $\bar{z}=z=z_{f}$. 
We next evaluate and integrate the Wess-Zumino and Solari-Kochetov terms in the tunnelling action. We denote these by $I_{\mathrm{WZ}}$ and $I_{\mathrm{SK}}$. Since the calculations are somewhat lengthy, it is best to do the two terms separately. We begin with $I_{\mathrm{WZ}}$, considering instanton 1, i.e., that given by (6.13) and (6.14). After some algebra, we obtain

$$
a_{\mathrm{WZ}}(\tau)=-\frac{\pi_{2}(\tanh t)}{\pi_{3}(\tanh t)} \frac{\omega}{2} \operatorname{sech}^{2} t
$$

where $\pi_{2}$ and $\pi_{3}$ are polynomials of degree 2 and 3 , whose explicit form we do not require. What we do need is the differential $a_{\mathrm{WZ}} d \tau$. If we make the substitution

$$
v=\tanh t
$$

and factorize the polynomials $\pi_{2}$ and $\pi_{3}$, we obtain

$$
\int_{-\infty}^{\infty} a_{\mathrm{WZ}}(\tau) d \tau=-\int_{-1}^{1} \frac{\left(v-v_{3}\right)\left(v-v_{4}\right)}{\left(v-v_{1}\right)\left(v-v_{2}\right)\left(v-v_{5}\right)} d v
$$

where

$$
\begin{aligned}
v_{1,2} & =\frac{1}{\sqrt{\lambda}}\left(\frac{1+\tilde{h}}{1-\tilde{h}}\right)^{1 / 2}(-1 \pm \sqrt{1-\lambda}), \\
v_{3,4} & =\frac{-\sqrt{1-\tilde{h}^{2}} \pm \sqrt{1-\tilde{h}^{2}-\lambda}}{\sqrt{\lambda}} \\
v_{5} & =-\frac{\sqrt{\lambda}}{\sqrt{1-\tilde{h}^{2}}} .
\end{aligned}
$$

The integral is best done by decomposing the integrand into partial fractions. We find

$$
\frac{\left(v-v_{3}\right)\left(v-v_{4}\right)}{\left(v-v_{1}\right)\left(v-v_{2}\right)\left(v-v_{5}\right)}=\frac{1}{v-v_{5}}+\frac{\beta}{v-v_{1}}-\frac{\beta}{v-v_{2}}
$$

where

$$
\beta=-\frac{\tilde{h}}{\sqrt{1-\lambda}}
$$

Thus,

$$
I_{\mathrm{WZ}}=j \int_{-\infty}^{\infty} a_{\mathrm{WZ}} d \tau=-j\left[\ln \left(\frac{1-v_{5}}{-1-v_{5}}\right)+\beta \ln \left(\frac{1-v_{1}}{-1-v_{1}}\right)-\beta \ln \left(\frac{1-v_{2}}{-1-v_{2}}\right)\right]
$$


The ratio involving $v_{5}$ is

$$
\frac{1-v_{5}}{-1-v_{5}}=\frac{\sqrt{\lambda}+\sqrt{1-\tilde{h}^{2}}}{\sqrt{\lambda}-\sqrt{1-\tilde{h}^{2}}} \equiv \tilde{R}_{1}
$$

while the $\beta$ terms combine to yield the logarithm of

$$
\frac{1-v_{1} v_{2}+\left(v_{2}-v_{1}\right)}{1-v_{1} v_{2}-\left(v_{2}-v_{1}\right)}=\frac{\tilde{h} \sqrt{\lambda}+\sqrt{1-\lambda} \sqrt{1-\tilde{h}^{2}}}{\tilde{h} \sqrt{\lambda}-\sqrt{1-\lambda} \sqrt{1-\tilde{h}^{2}}} \equiv \tilde{R}_{2} .
$$

Collecting together the various parts, we have

$$
I_{\mathrm{WZ}, 1}=-j \ln \tilde{R}_{1}+\frac{j \tilde{h}}{\sqrt{1-\lambda}} \ln \tilde{R}_{2} .
$$

We have added another suffix to show that this pertains to instanton 1.

The next step is to integrate the Solari-Kochetov term. For this we first need $\phi_{\mathrm{SK}}$. From Eqns. (6.4) and (3.2) we find,

$$
\begin{aligned}
\phi_{\mathrm{SK}}=\frac{\tilde{k}_{1} j}{2(1+\bar{z} z)^{2}}[ & -4\left(1-2 \bar{z} z-(\bar{z} z)^{2}\right)+2 \lambda\left((1+\bar{z} z)^{2}+2(\bar{z}-z)^{2}+\bar{z} z(\bar{z}-z)^{2}\right) \\
& \left.+4 \tilde{h}(1+\bar{z} z)^{2}\right] .
\end{aligned}
$$

(The reader may verify that as $\tau \rightarrow \pm \infty, \phi_{\mathrm{SK}} \rightarrow \omega_{1}$. This provides a check on our earlier calculation of $\omega_{1}$.) After a little more work, we find,

$$
\begin{aligned}
\phi_{\mathrm{SK}}-\omega_{1}= & \frac{\tilde{k}_{1} j}{(1+\bar{z} z)^{2}}\left[(1+\tilde{h})^{2}(1+\bar{z} z)^{2}+\lambda(\bar{z}-z)^{2}-4\right] \\
& +\tilde{k}_{1} j \lambda \frac{(\bar{z}-z)^{2}}{1+\bar{z} z} .
\end{aligned}
$$

We have written this expression in such a way that it is convenient to integrate the terms on the two lines separately. That is, we write,

$$
I_{\mathrm{SK}}=\frac{1}{2} \int_{-\infty}^{\infty}\left(\phi_{\mathrm{SK}}-\omega_{1}\right) d \tau=I_{A}+I_{B}
$$

where,

$$
\begin{aligned}
I_{A} & =\frac{1}{2} \tilde{k}_{1} j \int_{-\infty}^{\infty} d \tau \frac{(1+\tilde{h})^{2}(1+\bar{z} z)^{2}+\lambda(\bar{z}-z)^{2}-4}{(1+\bar{z} z)^{2}}, \\
I_{B} & =\frac{1}{2} \tilde{k}_{1} j \lambda \int_{-\infty}^{\infty} d \tau \frac{(\bar{z}-z)^{2}}{1+\bar{z} z} .
\end{aligned}
$$


It is to be understood that the integrands are evaluated along the instanton trajectories. We may exploit this fact to simplify the integrand for $I_{A}$ by using energy conservation and so eliminate the term in $\lambda$. When this is done, we obtain

$$
I_{A}=\tilde{k}_{1} j(1+\tilde{h}) \int_{-\infty}^{\infty} d \tau \frac{-(1-\tilde{h})+(1+\tilde{h}) \bar{z} z}{1+\bar{z} z}
$$

The integrals are evaluated in the same way as $I_{\mathrm{WZ}}$. With the same change of variables, and definitions of $v_{1}$ to $v_{5}$ as before, for $I_{A}$ we get

$$
\begin{aligned}
I_{A} & =-\frac{\left(1-\tilde{h}^{2}\right)^{1 / 2}(1+\tilde{h})}{\sqrt{\lambda}(1-\tilde{h})} \int_{-1}^{1} \frac{d v}{\left(v-v_{1}\right)\left(v-v_{2}\right)} \\
& =-\frac{(1+\tilde{h})}{2 \sqrt{1-\lambda}} \int_{-1}^{1}\left[\frac{1}{v-v_{1}}-\frac{1}{v-v_{2}}\right] d v \\
& =-\frac{(1+\tilde{h})}{2 \sqrt{1-\lambda}} \ln \tilde{R}_{2} .
\end{aligned}
$$

Likewise, for $I_{B}$ we get

$$
I_{B}=-\frac{1}{2} \int_{-1}^{1} \frac{(v-1)(v+1)}{\left(v-v_{1}\right)\left(v-v_{2}\right)\left(v-v_{5}\right)} d v
$$

The partial fraction decomposition yields

$$
\frac{(v-1)(v+1)}{\left(v-v_{1}\right)\left(v-v_{2}\right)\left(v-v_{5}\right)}=\frac{1}{v-v_{5}}+\frac{\beta^{\prime}}{v-v_{1}}-\frac{\beta^{\prime}}{v-v_{2}}
$$

where

$$
\beta^{\prime}=-(1-\lambda)^{-1 / 2}
$$

Hence,

$$
I_{B}=-\frac{1}{2} \ln \tilde{R}_{1}+\frac{1}{2 \sqrt{1-\lambda}} \ln \tilde{R}_{2}
$$

Thus the Solari-Kochetov contribution to the action for instanton 1 is

$$
I_{\mathrm{SK}}=-\frac{1}{2} \ln \tilde{R}_{1}-\frac{\tilde{h}}{2 \sqrt{1-\lambda}} \ln \tilde{R}_{2}
$$

Note that this is $O(1 / j)$ relative to the Wess-Zumino contribution. Adding together the two contributions, we obtain the total action 


$$
I=-\left(j+\frac{1}{2}\right) \ln \tilde{R}_{1}+\frac{j h}{\sqrt{1-\lambda}} \ln \tilde{R}_{2}
$$

In the second term we have used the formula $\left(j-\frac{1}{2}\right) \tilde{h}=j h$.

We now turn to the prefactor $P$. In evaluating this, we may ignore differences of order $1 / j$, i.e., we may replace $\tilde{\jmath}$ by $j, \tilde{h}$ by $h$, etc. The quantity consists of a product of various factors, all of which are already available. Thus,

$$
\frac{j}{\pi N}=\frac{j}{\pi\left(1+z_{0}^{2}\right)^{2}}
$$

The factors $\bar{\zeta}_{+}$and $\zeta_{-}$are found by differentiating the formulas (6.13) and (6.14) and examining the limits $\tau \rightarrow \pm \infty$. In this we way we get

$$
\begin{aligned}
\zeta_{-} & =-2 z_{0}, \\
\bar{\zeta}_{+} & =2 z_{0} \frac{\sqrt{1-h^{2}}-\sqrt{\lambda}}{\sqrt{1-h^{2}}+\sqrt{\lambda}} .
\end{aligned}
$$

Finally,

$$
\frac{2 \omega}{\omega_{1}-\omega}=4 \frac{\sqrt{\lambda\left(1-h^{2}\right)}}{\left(1-h^{2}+\lambda\right)-2 \sqrt{\lambda\left(1-h^{2}\right)}}=4 \frac{\sqrt{\lambda\left(1-h^{2}\right)}}{\left[\sqrt{1-h^{2}}-\sqrt{\lambda}\right]^{2}}
$$

Making use of the identity

$$
\frac{2 z_{0}}{1+z_{0}^{2}}=\left(1-h^{2}\right)^{1 / 2}
$$

we obtain

$$
P=-\frac{4 j}{\pi} \frac{\lambda^{1 / 2}\left(1-h^{2}\right)^{3 / 2}}{1-h^{2}-\lambda}
$$

We can now obtain the contribution of instanton 1 to the tunnelling amplitude by substituting Eqs. (6.47) and (6.53) in the general formula (4.10). Denoting this quantity by $\Delta_{1}$, we have

$$
\Delta_{1}=2 \omega \sqrt{|P|} e^{I-i \pi / 2}
$$

where the additional factor of $e^{-i \pi / 2}$ arises from the fact that $P<0$. 
It remains to obtain the tunnelling amplitude $\Delta_{2}$ from the second instanton. Because the two instantons are related by a complex shift in $t$, it is apparent that the actions $I_{1,2}$ (where we temporarily add suffixes to distinguish the two) and the prefactors $P_{1,2}$ will be given by the same analytic expressions. However, the phases to be assigned to the actions and $\sqrt{P}$ are somewhat ambiguous. Unlike the case of a particle moving in one dimension, the prefactor in the general formula does not arise as the determinant of a Hermitean quadratic form, and there is no unambiguous way for factors of $i$ to get partitioned between the prefactor and the exponent. The surest way of fixing the relative phases is to appeal to a physical argument. Alternatively, this can be regarded as fixing the signs of the amplitudes $a_{i}$ and $a_{f}$.

For the $\mathrm{Fe}_{8}$ Hamiltonian (6.4), let us work in the $J_{z}$ basis $|j, m\rangle$ with the standard definition of the raising and lowering operators $J_{ \pm}$, so that the matrix elements $\left\langle j, m \pm 1\left|J_{ \pm}\right| j, m\right\rangle$ are all real. Then the matrix of $\hat{H}$ is completely real, and since it is Hermitean, all its eigenvalues and eigenvectors are also real. Secondly, since $z_{i}=z_{0}$ and $z_{f}=-z_{0}$ are real, the states $\left|z_{i, f}\right\rangle$ are real, i.e., all the matrix elements $\left\langle j, m \mid z_{i, f}\right\rangle$ are real. Thus the amplitudes $a_{i}$ and $a_{f}$ are real. It follows that the amplitude $K$ is real, and so is the one-instanton contribution to it, i.e., $\Delta_{1}+\Delta_{2}$ is real. Therefore, we must have

$$
\Delta_{2}=\Delta_{1}^{*}
$$

Equation (6.55) determines $\Delta_{2}$, and the energy splitting $\Delta$ completely. However, it is still useful to investigate the origin of the phase difference in the actions a little more closely. As readers will have noticed already, the integrand in Eq. (6.25) is singular at $v=v_{2}$ and $v=v_{5}$, since for $\tilde{h}<\sqrt{1-\lambda}$,

$$
v_{1}<-1, \quad-1<v_{2}<1, \quad-1<v_{5}<1 \text {. }
$$

Correspondingly, both $\tilde{R}_{1}$ and $\tilde{R}_{2}$ are negative, and both $\ln \tilde{R}_{1}$ and $\ln \tilde{R}_{2}$ must be interpreted to have an imaginary part of $\pi$ modulo an integer multiple of $2 \pi$. The question is what the assignment should be for the two instantons. We can see this most easily by examining the 
difference $\Delta I_{\mathrm{WZ}}=I_{\mathrm{WZ}, 2}-I_{\mathrm{WZ}, 1}$. To this end, we note that the WZ one-form may be written as a complex one-form in the $z$ plane,

$$
a_{\mathrm{WZ}} d \tau=\frac{1}{1+z \bar{z}(z)}\left[z \frac{d \bar{z}}{d z}-\bar{z}(z)\right] d z \equiv F(z) d z
$$

with $\bar{z}(z)$ given by Eq. (6.11). Thus, $I_{\mathrm{Wz}}$ may be written as a $z$-plane contour integral of $F(z)$ from $z_{0}$ to $-z_{0}$. In fact, apart from a scale factor of $z_{0}$, the substitution (6.24) is tantamount to changing the integration variable to $z$, so we see that $F(z)$ has poles at $z_{0} v_{2}$ and $z_{0} v_{5}$ (the one at $z_{0} v_{1}$ does not matter). The two instantons go around these poles in opposite senses, so $\Delta I_{\mathrm{WZ}}$ is given by integrating $F(z)$ along a closed contour from $z_{0}$ to $-z_{0}$ and back to $z_{0}$ :

$$
\Delta I_{\mathrm{WZ}}=\oint F(z) d z
$$

The residues at the poles can be read off the partial fraction decomposition (6.29), yielding

$$
I_{\mathrm{WZ}, 2}-I_{\mathrm{WZ}, 1}=2 j \pi-\frac{2 j \tilde{h} \pi}{\sqrt{1-\lambda}} .
$$

This is precisely what we would obtain from Eq. (6.55), for that would have us assign $\pm i \pi$ for $\ln \tilde{R}_{1}$ (and $\ln \tilde{R}_{2}$ ) for the two instantons.

The energy splitting is given by

$$
\Delta=\Delta_{1}+\Delta_{2}^{*}
$$

To compare with previous results, it is useful to rewrite this as follows. Consider the real part of the action,

$$
\Gamma_{0}=-\operatorname{Re} I=\left(j+\frac{1}{2}\right) \ln \left|\tilde{R}_{1}\right|-\frac{j h}{\sqrt{1-\lambda}} \ln \left|\tilde{R}_{2}\right|
$$

The ratios $\tilde{R}_{1}$ and $\tilde{R}_{2}$ are defined in terms of the field $\tilde{h}$. If we write $\tilde{h}=h+O(1 / j)$, and expand in powers of $1 / j$, we discover that

$$
\Gamma_{0}=\left(j+\frac{1}{2}\right) \ln \left|R_{1}\right|-\frac{j h}{\sqrt{1-\lambda}} \ln \left|R_{2}\right|+O\left(j^{-1}\right),
$$


where $R_{i}$ is obtained from $\tilde{R}_{i}$ by simply deleting the tildes above the $h$ 's. Note that the corrections are of $O(1 / j)$, not $O(1)$. These are beyond the accuracy to which we are working, so we simply drop them henceforth.

Thus, the complete expression for the splitting is

$$
\Delta=\sqrt{\frac{8}{\pi}} \omega F^{1 / 2} e^{-\Gamma_{0}} \cos \Lambda .
$$

We give the expressions for $F, \Gamma_{0}$ and $\Lambda$ for ready reference:

$$
\begin{aligned}
F & =8 j \frac{\lambda^{1 / 2}\left(1-h^{2}\right)^{3 / 2}}{1-h^{2}-\lambda} \\
\Gamma_{0} & =\left(j+\frac{1}{2}\right) \ln \left[\frac{\sqrt{1-h^{2}}+\sqrt{\lambda}}{\sqrt{1-h^{2}}-\sqrt{\lambda}}\right]-\frac{j h}{\sqrt{1-\lambda}} \ln \left[\frac{\sqrt{(1-\lambda)\left(1-h^{2}\right)}+h \sqrt{\lambda}}{\sqrt{(1-\lambda)\left(1-h^{2}\right)}-h \sqrt{\lambda}}\right] \\
\Lambda & =\operatorname{Im} I-\frac{\pi}{2} \\
& =j \pi\left(1-\frac{h}{\sqrt{1-\lambda}}\right) .
\end{aligned}
$$

Our answer for $\Delta$ is identical to that found by means of the discrete WKB method in [19] [see Eqs. (5.1-5.5)]. Naturally, the points at the which the tunnel splitting vanishes are the same too.

The nontrivial aspect of this calculation is that there are $1 / j$ corrections in the quenching condition. If we simply take the energy expectation $H(\bar{z}, z)=\langle z|\hat{H}| z\rangle /\langle z \mid z\rangle$ in the WessZumino term, we have the problem that the anisotropy and field terms scale with $j$ differently if $1 / j$ corrections are included. This is how the quenching condition was found in [5], but the $1 / j$ corrections were never considered, so it was some what serendipitous that the condition that was stated turned out to be rigorously correct. By including the SK correction, this deficiency is now repaired.

\section{CONCLUSION}

We have seen that, once the extra-phase contribution is included, the coherent-state path integral for spin provides an accurate and effective tool for calculating tunnel splitting. In 
particular the Weyl shift $j \rightarrow j+\frac{1}{2}$ appears automatically. It must therefore be possible to put the spin coherent-state path integral on the same sound mathematical footing as the conventional Feynman integral.

\section{ACKNOWLEDGMENTS}

Work at Urbana and Evanston was supported by the National Science Foundation under grants DMR-98-17941 and DMR-9616749, respectively. EK thanks the Russian Foundation for Fundamental Research for supporting this work through Grant $N_{\underline{O}}$ 00-01-00049.

\section{APPENDIX}

Here we derive equation (4.6). We first apply an $S U(2)$ rotation to

$$
H_{\text {initial }}(\bar{z}, z)=\frac{2 j}{\left(1+z_{i}^{*} z_{i}\right)^{2}}\left[\omega_{1}\left(z-z_{i}\right)\left(\bar{z}-z_{i}^{*}\right)+\frac{1}{2} \omega_{2}\left(z-z_{i}\right)^{2}+\frac{1}{2} \omega_{2}^{*}\left(\bar{z}-z_{i}^{*}\right)^{2}\right]
$$

in order to place $z_{i}, \bar{z}_{i}$ at the origin, and to make the coefficient $\omega_{2}$ real. The result is

$$
H(\bar{z}, z)=2 j\left[\omega_{1} \bar{z} z+\frac{1}{2} \omega_{2} z^{2}+\frac{1}{2} \omega_{2} \bar{z}^{2}\right]
$$

In the semiclassical limit, $2 j \gg 1$, we may ignore the curvature of the phase space and, after rescaling $\sqrt{2 j} z \rightarrow z$ to account for the difference in the coefficient in the kinetic terms,

identify $H(\bar{z}, z)$ with the coherent state classical Hamiltonian for the squeezed harmonic oscillator

$$
\hat{H}=\omega_{1} a^{\dagger} a+\frac{1}{2} \omega_{2}\left(a^{\dagger^{2}}+a^{2}\right)
$$

The Bogoliubov transformation

$$
\begin{aligned}
b & =\cosh \theta a+\sinh \theta a^{\dagger} \\
b^{\dagger} & =\sinh \theta a+\cosh \theta a^{\dagger}
\end{aligned}
$$

reduces the Hamiltonian 


$$
\hat{H}_{\text {squeezed }}=\Omega \cosh 2 \theta\left(a^{\dagger} a+\frac{1}{2}\right)+\frac{1}{2} \Omega \sinh 2 \theta\left(a^{\dagger^{2}}+a^{2}\right)
$$

to

$$
\hat{H}_{\text {squeezed }}=\Omega\left(b^{\dagger} b+\frac{1}{2}\right)
$$

and so we identify

$$
\begin{aligned}
\Omega=\omega & =\sqrt{\omega_{1}^{2}-\omega_{2}^{2}}, \\
\Omega \cosh 2 \theta & =\omega_{1}, \\
\Omega \sinh 2 \theta & =\omega_{2} .
\end{aligned}
$$

The eigenvalues of $\hat{H}$ are therefore

$$
E_{n}=\omega\left(n+\frac{1}{2}\right)-\frac{1}{2} \omega_{1}
$$

The operators $a^{\dagger} a, a^{2}$ and $a^{\dagger^{2}}$ generate the Lie algebra $s u(1,1)$. Therefore either the flat phase-space coherent state path integral or standard $s u(1,1)$ disentangling methods [20,21] can be used to derive

$$
\left\langle\zeta_{f}\left|e^{-\hat{H} T}\right| \zeta_{i}\right\rangle=D^{-\frac{1}{2}} \exp \left\{D^{-1}\left(\bar{\zeta}_{f} \zeta_{i}-\frac{1}{2} \sinh 2 \theta \sinh \omega T\left(\bar{\zeta}_{f}^{2}+\zeta_{i}^{2}\right)\right)\right\} e^{-\frac{1}{2} \omega_{1} T}
$$

where

$$
D=e^{\omega T} \cosh ^{2} \theta-e^{-\omega T} \sinh ^{2} \theta
$$

and the harmonic oscillator coherent states $|\zeta\rangle$ are defined by

$$
|\zeta\rangle=\exp \zeta a^{\dagger}|0\rangle, \quad a|0\rangle=0
$$

In the large $-T$ limit, and with $\zeta_{i}$ and $\bar{\zeta}_{f}$ both at the origin, this reduces to

$$
\left\langle 0\left|e^{-\hat{H} T}\right| 0\right\rangle \rightarrow(\cosh \theta)^{-1} e^{-\frac{1}{2}\left(\omega-\omega_{1}\right) T}=\sqrt{\frac{2 \omega}{\omega+\omega_{1}}} e^{-\frac{1}{2}\left(\omega-\omega_{1}\right) T} .
$$

We now rotate back to the original $z_{i}$. Taking note of the transformation properties of the $|z\rangle$ 's, we get

$$
K_{i}=\left(1+\bar{z}_{i} z_{i}\right)^{2 j} \sqrt{\frac{2 \omega}{\omega+\omega_{1}}} e^{-\frac{1}{2}\left(\omega-\omega_{1}\right) T}
$$

as claimed. 


\section{REFERENCES}

[1] W. Wernsdorfer and R. Sessoli, Science 284 (1999) 133.

[2] E. M. Chudnovsky, L. Gunther, Phys. Rev. Lett. 60 (1988) 661.

[3] D. Loss, D. P. Vincenzo, G. Grinstein, Phys. Rev. Lett. 69 (1992) 3232.

[4] J. von Delft and C. L. Henley, Phys. Rev. Lett. 69 (1992) 3236.

[5] A. Garg, Europhys. Lett. 22 (1993) 205.

[6] H. Kuratsuji, Y. Mizobuchi, J. Math. Phys. 22 (1981) 757.

[7] A. Garg, G-H. Kim, Phys. Rev. B 45 (1992) 921.

[8] M. Enz, R. Schilling, J. Phys. C 19 (1986) L711; 1765.

[9] V. I. Belinicher, C. Providencia, J. da Providencia, J. Phys. A 30 (1997) 5633.

[10] H. G. Solari, J. Math. Phys. 28 (1987) 1097.

[11] E. A. Kochetov, J. Math. Phys. 36 (1995) 4667.

[12] V. R. Vieira, P. D. Sacramento Nucl. Phys. B 448 (1995) 331.

[13] M. Stone, K-S. Park, A. Garg, J. Math. Phys. 41 (2000) 8025.

[14] H. J. Lipkin, N. Meshkov A.J. Glick. Nucl. Phys. 62 (1965) 188.

[15] A. Perelomov, Generalized Coherent States and their Applications, (Springer-Verlag, Berlin 1986).

[16] M. B. Lowe, M. Stone, Nucl. Phys. B136 (1978) 177.

[17] J. C. Collins, D. E. Soper Annals of Physics, 112 (1978) 209.

[18] A. Garg J. Math. Phys. 39 (1998) 5166.

[19] A. Garg, Phys. Rev. B 64, 094413 (2001); [see Eqs. (5.1-5.5)] 
[20] H. P. Yuen, Phys. Rev. A 13 (1976) 2226.

[21] D. Stoler, Phys. Rev. D 1 (1970) 3217; Phys. Rev. D 2 (1971) 1925 (erratum). 